\title{
A Note on Small Linear-Ordering Polytopes
}

\author{
Gerhard Reinelt \\ Institut für Angewandte Mathematik, Universität Heidelberg, \\ Im Neuenheimer Feld 293, W-6900 Heidelberg, Federal Republic of Germany \\ reinelt@ares.iwr.uni-heidelberg.de
}

\begin{abstract}
In this paper we discuss the polyhedral structure of polytopes associated with the linear-ordering problem. We give explicit lists of facets of small linearordering polytopes for complete digraphs on up to seven nodes. For the latter we give a description that we believe to be complete.
\end{abstract}

\section{Introduction and Basic Results}

Suppose $m$ persons have assessed $n$ objects $O_{1}, \ldots, O_{n}$ by pairwise comparisons (i.e., for every pair of objects $O_{i}$ and $O_{j}$ we know how many persons prefer object $O_{i}$ to $O_{j}$, object $O_{j}$ to $O_{i}$, respectively) and that based on these judgments a ranking of the objects is to be found. One possibility is to determine a linear ordering of the objects such that the number of individual pairwise assessments that are not in accordance with this ordering is minimized. This problem is known as the linear-ordering problem and belongs to the class of $\mathcal{N} \mathscr{P}$-hard combinatorial optimization problems.

The linear-ordering problem has several practical applications in economics, scheduling, sports, archeology, and social sciences. The most successful algorithm to solve linear-ordering problems to optimality is given in Grötschel et al. (1984). It is a branch and cut method which exploits knowledge about the so-called linear-ordering polytope. This present paper is concerned with some new results about the structure of this polytope, in particular we address the question of characterizing small instances completely.

In what follows we use a graph-theoretical framework to deal with the linear-ordering problem. Let $D_{n}=\left(V_{n}, A_{n}\right)$ be the complete digraph on $n$ nodes, i.e., the directed graph with node set $V_{n}=\{1,2, \ldots, n\}$ and the property that, for every pair of nodes $i$ and $j$, there is an arc from $i$ to $j$ and an arc from $j$ to $i$. A tournament (or spanning tournament) $T$ in $A_{n}$ consists of a subset of arcs 
containing, for every pair of nodes $i$ and $j$, either $\operatorname{arc}(i, j)$ or arc $(j, i)$ but not both. A (spanning) acyclic tournament is a tournament without directed cycles, i.e., not containing an arc set of the form $\left\{\left(v_{1}, v_{2}\right),\left(v_{2}, v_{3}\right), \ldots,\left(v_{k}, v_{1}\right)\right\}$ for some $k>1$ and nodes $v_{1}, v_{2}, \ldots, v_{k}$. It is easy to see that such an acyclic tournament corresponds to a linear ordering of the nodes and vice versa: the first node is the one without entering arcs in $T$, the second node is the one with one entering arc, etc., and the node ranked lowest is the one without leaving arcs in $T$. We denote the linear ordering that ranks node $v_{i_{1}}$ first, $v_{i_{2}}$ second, etc., by $\left\langle v_{i_{1}}, v_{i_{2}}, \ldots, v_{i_{n}}\right\rangle$.

To be able to apply methods from linear programming we associate with every subset $B$ of $A_{n}$ an incidence vector $\chi^{B}$. The incidence vector has $n(n-1)$ components indexed by the arcs of $A_{n}$ and is defined by

$$
\chi_{i j}^{B}= \begin{cases}1 & \text { if }(i, j) \in B, \\ 0 & \text { otherwise. }\end{cases}
$$

A polyhedron is defined as the intersection of finitely many half-spaces or equivalently as the solution set of a finite system of linear inequalities and equations. A polytope is a bounded polyhedron. The minimal (with respect to set inclusion) nonempty proper faces of a polyhedron are called vertices, the maximal proper faces are its facets. A polytope can be defined as the convex hull of its vertices. We use this alternative to define the linear-ordering polytope.

Definition 1.1. Let $D_{n}=\left(V_{n}, A_{n}\right)$ be the complete digraph on $n$ nodes and $m=n(n-1)$. Then the linear-ordering polytope $P_{\text {Lo }}^{n}$ is defined as the convex hull of the incidence vectors of the acyclic tournaments in $D_{n}$, i.e.,

$$
P_{\mathrm{LO}}^{n}:=\operatorname{conv}\left\{\chi^{T} \in\{0,1\}^{m} \mid T \subset A_{n} \text { is an acyclic tournament }\right\} .
$$

It is immediate that the vertices of $P_{\text {Lo }}^{n}$ correspond to acyclic tournaments and vice versa. Moreover, solving the linear-ordering problem now in principle amounts to maximizing a linear objective function over this polytope, where an objective function coefficient $c_{i j}$ is associated with every arc $(i, j)$. In our example $c_{i j}$ would be the number of comparisons ranking object $O_{i}$ before object $O_{j}$.

For algorithmic purposes a description of the polytope as convex hull of vertices is of no help. Instead we are interested in its facial structure, i.e., in a description by systems of linear equations $D x=d$ and linear inequalities $A x \leq b$ such that $P_{\text {LO }}^{n}=\left\{x \in \mathbf{R}^{n} \mid D x=d, A x \leq b\right\}$. In addition we are interested in finding a minimal equation system and a nonredundant system of inequalities. It is known that in such a system of inequalities every inequality corresponds to a facet.

The facial structure of $P_{\text {Lo }}^{n}$ has been investigated in Grötschel $e t$ al. (1985) and Reinelt (1985). It is far beyond the scope of such investigations to derive a complete general description of the linear-ordering polytope. No such description is known for polytopes associated with $\mathscr{N} \mathscr{P}$-hard combinatorial optimization problems and, for complexity reasons, it is not likely that complete characterizations will ever be 
found. It turned out, however, that partial knowledge about the facial structure is already very effective for practical computations. The algorithm mentioned in the outset is based on such a partial description and was able to increase the size of solvable linear-ordering problems substantially.

Fairly recently further research on the polyhedral structure of $P_{\mathrm{LO}}^{n}$ has been undertaken. New facet-defining inequalities were given in Leung and Lee (1990) and Suck (1991). The paper by Fishburn (1991) gives a status report on the knowledge about $P_{\text {LO }}^{n}$. This report is of particular interest since it documents that two different lines of (almost) independent research concerning this polytope existed, one coming from combinatorial optimization and graph theory, and the other from the study of binary choice probabilities. Motivated by these recent developments we took another look at $P_{\mathrm{Lo}}^{n}$.

As well as identifying classes of facet-defining inequalities, there has always been interest in deriving complete linear descriptions for polytopes associated with small instances of combinatorial optimization problems. Though real problems are usually large scale, it is worthwhile investigating small polytopes. Facets derived for small polytopes can give hints for generalizations to facets for larger polytopes and studying small polytopes can also yield information on the relative importance of the different classes of facets. Our main interest was to consider the linear description of $P_{\mathrm{LO}}^{7}$ and possibly to identify new classes of facet-defining inequalities.

Some basic general properties of the linear-ordering polytope are easily derived.

Theorem 1.2. Let $n \geq 2$. Then the system

$$
x_{i j}+x_{j i}=1 \quad \text { for all } i, j \in V_{n}, \quad i<j,
$$

is a minimal equation system for $P_{\text {Lo. }}^{n}$

$P_{\text {LO }}^{n}$ is contained in the $(n \cdot(n-1))$-dimensional Euclidean space and hence the minimal equation system determines its dimension to be $n(n-1) / 2$. Since $P_{\text {Lo }}^{n}$ is not full-dimensional, a facet-defining inequality can be represented in different ways. However, due to the simple structure of the equation system, we can define a standard representation of inequalities which are valid for $P_{\text {LO. }}^{n}$.

Corollary 1.3. For every facet of $P_{\mathrm{Lo}}^{n}$ there exists an inequality $a^{T} x \leq \alpha$ defining it such that a has nonnegative coefficients and the property that, for every pair of nodes $i, j \in V_{n}$, at least one of the coefficients $a_{i j}$ or $a_{j i}$ is equal to zero.

We can use this observation to define a normal form for facet-defining inequalities. Namely, every facet can be represented uniquely by an inequality $a^{T} x \leq \alpha$ such that all coefficients $a_{i j}$ are nonnegative coprime integers and, moreover, we have $a_{i j} \cdot a_{j i}=0$ for every pair of nodes $i, j \in V_{n}$.

An important general question is to decide whether or not two facet-defining inequalities define the same facet, i.e., whether they are equivalent. The following 
theorem gives a sufficient condition for the nonequivalence of two inequalities which define facets of the linear-ordering polytope.

Theorem 1.4. Let $a^{T} x \leq \alpha$ and $b^{T} x \leq \beta$ be facet-defining inequalities for $P_{\text {Lo }}^{\text {n, }}$ $n \geq 2$, given in normal form. If there exists an arc $(i, j) \in A_{n}$ with $a_{i j}>0$ and $b_{i j}=0$ (or $b_{i j}>0$ and $a_{i j}=0$ ), then the inequalities define different facets.

The theorem does not exclude that two nonequivalent facet-defining inequalities have the same support. Indeed, such cases exist (see Leung and Lee, 1990; Suck, 1991). Two useful general properties of facet-defining inequalities for $P_{\text {Lo }}^{n}$ are stated in the following two lemmas.

Lemma 1.5 (Trivial Lifting Lemma). Let $a^{T} x \leq \alpha$ be a facet-defining inequality for $P_{\mathrm{LO}}^{n}, n \geq 2$. Define $\bar{a} \in \mathbf{R}^{(n+1) n}$ by setting $\bar{a}_{i j}=a_{i j}$, for all $(i, j) \in A_{n}$, and $\bar{a}_{i, n+1}=$ $\bar{a}_{n+1, i}=0$, for $i=1, \ldots, n$. Then $\bar{a}^{T} x \leq \alpha$ defines a facet of $P_{\mathrm{LO}}^{n+1}$.

Lemma 1.6 (Arc Reversal Lemma). Suppose $a^{T} x \leq \alpha$ is a facet-defining inequality for $P_{\mathrm{LO}}^{n}, n \geq 2$. If $b \in \mathbf{R}^{n(n-1)}$ is defined by $b_{i j}=a_{j i}$ for all $(i, j) \in A_{n}$, then $b^{T} x \leq \alpha$ is facet defining for $P_{\mathrm{LO}}^{\mathrm{n}}$.

We therefore know that the linear-ordering polytope $P_{\text {Lo }}^{n}$ inherits all facets from $P_{\mathrm{LO}}^{n-1}$ and that reversing the arcs in the support of a facet-defining inequality yields a new facet-defining inequality.

\section{Linear-Ordering Polytopes on at most Six Nodes}

The simplest class of valid inequalities for $P_{\text {Lo }}^{n}$ are the trivial inequalities $0 \leq x_{i j} \leq 1$ for all $(i, j) \in A_{n}$. They are also called hypercube constraints and are always valid for polytopes defined as the convex hull of $0-1$-vertices. Trivial inequalities define facets of $P_{\mathrm{LO}}^{n}$ for all $(i, j) \in A_{n}$. No two of the inequalities $x_{i j} \leq 1$ are equivalent. An inequality $-x_{i j} \leq 0$ reads $x_{j i} \leq 1$ in normal form and therefore does not give a further facet. The minimal equation system and the trivial inequalities are sufficient for describing $P_{\text {Lo }}^{2}$. Further inequalities are needed for $n \geq 3$.

The most natural inequalities to be taken into account in addition for a description of $P_{L O}^{n}$ are those which exclude incidence vectors of tournaments containing a dicycle. In the case of a dicycle $C$ of length 3 (i.e., consisting of three nodes) the corresponding inequality $\sum_{(i, j) \in C} x_{i j} \leq 2$ defines a facet of $P_{\text {Lo }}^{n}$ for $n \geq 3$ and we have $2 \cdot(n(n-1) / 3)$ different 3-dicycle inequalities which are nonequivalent. Inequalities associated with dicycles on more than three nodes do not define facets of $P_{\text {Lo. }}^{n}$.

A minimal equation system, trivial inequalities, and 3-dicycle inequalities are sufficient to completely describe $P_{\text {LO }}^{3}, P_{\text {LO }}^{4}$, and $P_{\text {Lo }}^{5}$. Hence their respective number of facets is 8,20 , and 40 . Explicit proofs can be found in, e.g., Dridi 
(1980) and Fishburn (1990). We denote by $P_{\mathrm{C}}^{n}$ the polytope defined by the minimal equation system and the facet-defining inequalities derived so far. In earlier publications it was believed that $P_{\text {LO }}^{n}=P_{\mathrm{C}}^{n}$ (see De Cani, 1969; Bowman, 1972), but this is not the case.

In Reinelt (1985) a partial description of $P_{\mathrm{LO}}^{6}$ was given. It consisted of the 15 equations forming the minimal equation system, 30 trivial inequalities, 403 -dicycle inequalities, 1203 -fence inequalities (see $F_{3}$ of Fig. 4.1), and 720 particular Möbius-ladder inequalities on six nodes (see $F_{4}$ of Fig. 4.1).

Using a computer program (some details of which are given below) to derive the linear description of the convex hull of a given number of points, we could verify that this description is indeed complete for $P_{\mathbf{L O}}^{6}$.

Therefore, a complete description of $P_{L O}^{6}$ is given by the minimal equation system and 910 facet-defining inequalities.

\section{The Computation Technique for $\boldsymbol{P}_{\mathrm{LO}}^{7}$}

No attempt to give an explicit description of $P_{\text {Lo }}^{7}$ has been made so far in the literature. We used a computer program (Christof, 1991) to try to achieve this. The program for transforming the representation of a polytope as a convex hull of points into the representation as intersection of half-spaces is based on Fourier-Motzkin elimination. In order to keep the number of inequalities small it was enhanced by a redundancy check due to Tschernikov (1971). Rational arithmetic is used to guarantee exact numerical results.

The linear-ordering polytope on seven nodes has 5040 vertices. Due to storage and CPU time limits direct application of the computer code was not possible. We therefore proceeded along the same lines as Christof et al. (1991), where complete descriptions of small traveling salesman polytopes were considered. The linear-ordering polytope is symmetric, i.e., the facets containing one specific vertex can be obtained from the facets containing some arbitrary vertex by a suitable permutation of the nodes.

If we denote by $x_{1}, x_{2}, \ldots, x_{5040}$ the incidence vectors corresponding to linear orderings of seven nodes, then it is sufficient to consider

$$
\operatorname{conv}\left\{x_{1}\right\}+\operatorname{cone}\left\{x_{2}-x_{1}, x_{3}-x_{1}, \ldots, x_{5040}-x_{1}\right\} .
$$

From a linear description of this cone we can obtain the complete description of $P_{\text {Lo. }}^{7}$. Of course, it is sufficient to only take those directions $x_{i}-x_{1}$ into account where $x_{i}$ is a vector adjacent to $x_{1}$.

To identify adjacent vertices on the linear-ordering polytope we make use of a result stated in Young (1978) (see also Hausmann, 1980). To formulate this theorem we make some conventions. If $x$ and $y$ are incidence vectors of spanning acyclic tournaments in $D_{n}$, then we denote by $x \cup y(x \cap y)$ the vector obtained by calculating the logical "or" ("and"). By $x \oplus y$ we denote the vector whose components are 1 if the corresponding components of $x$ and $y$ are different and 0 otherwise ("exclusive or"). Note that $x \cap y$ induces a partial ordering of the nodes of $D_{n}$. 
Theorem 3.1. Let $T_{1}$ and $T_{2}$ be two distinct linear orderings of the nodes of $D_{n}$. Construct a graph $\Gamma\left(T_{1}, T_{2}\right)=\left(V_{\Gamma}, E_{\Gamma}\right)$ as follows:

$$
\begin{aligned}
& V_{\Gamma}=\left\{\{u, v\} \mid z_{u v}=1, \text { where } z=\chi^{T_{1}} \oplus \chi^{T_{2}}\right\} \\
& E_{\Gamma}=\left\{(\{u, v\},\{v, w\}) \mid z_{u w}=1 \text { or } z_{w u}=1, \text { where } z=\chi^{T_{1}} \cap \chi^{T_{2}}\right\}
\end{aligned}
$$

Then $\chi^{T_{1}}$ and $\chi^{T_{2}}$ are adjacent with respect to $P_{\mathrm{Lo}}^{n}$ if and only if the graph $\Gamma\left(T_{1}, T_{2}\right)$ is connected.

The adjacency criterion can be efficiently checked and it turns out that the number of 5039 directions can be reduced to 1042. Still the program failed to compute the linear description. To reduce the amount of work further we considered the cones

$$
\operatorname{conv}\left\{x_{1}\right\}+\operatorname{cone}\left\{y_{1}, \ldots, y_{1042}\right\}+\operatorname{cone}\left\{-y_{i}\right\}
$$

where $y_{i}$ are the edges from $x_{1}$ to its adjacent vertices.

In the case of the traveling salesman polytope on eight nodes we could make use of symmetry considerations at this point. This enabled us to reduce the number of cones to be considered there from 730 to 59 . We were not able to identify symmetry classes in the case of the linear-ordering polytope. Since computation of the linear description of one such cone as above takes on average about 2 days on a SUN SPARCstation $1+$ we confined ourselves to a reasonable subset of the 1042 cones. If $x_{1}$ is the incidence vector corresponding to the canonical ordering $\langle 1,2,3,4,5,6,7\rangle$, then we considered the six cones arising when $y_{i}, i=1, \ldots, 6$, are the directions to the following six adjacent vertices of $x_{1}$ :

$$
\begin{aligned}
& \langle 2,1,3,4,5,6,7\rangle, \\
& \langle 1,3,2,4,5,6,7\rangle, \\
& \langle 1,2,4,3,5,6,7\rangle, \\
& \langle 1,2,3,5,4,6,7\rangle, \\
& \langle 1,2,3,4,6,5,7\rangle, \\
& \langle 1,2,3,4,5,7,6\rangle .
\end{aligned}
$$

This way we can identify all facets with the property that they contain two vertices corresponding to linear orderings which just differ in two adjacent positions.

\section{The Linear Description of $\boldsymbol{P}_{\text {LO }}^{7}$}

We now discuss in detail the classes of facets of $P_{\text {Lo }}^{7}$ that we could identify. Every class is represented by a digraph $F=(W, B)$ with certain arc weights $a_{i j}$ for every arc $e \in B$ and a right-hand side $\alpha$ such that $\sum_{(i, j) \in B} a_{i j} x_{i j} \leq \alpha$ defines a facet of $P_{\mathrm{LO}}^{7}$. 
We say that a class is invariant (with respect to arc reversal) if the digraph $F^{*}$ obtained from $F$ by reversing all arcs (keeping the arc weights) is isomorphic to $F$.

There are 19 different classes of facets whose corresponding digraphs (except for the trivial inequalities) are shown in Fig. 4.1. Arc weights not listed explicitly are 1, the right-hand side of the associated inequality is given in Table 4.1. Classes are named $F_{1}-F_{19}$. We give some comments on these classes.

$F_{1}$. These are the trivial inequalities. The class is invariant.

$F_{2}$. The second class consists of 3-dicycles whose associated inequalities were the first known nontrivial facet-defining inequalities. Arc reversal does not create new digraphs.

$F_{3}$. This class contains 3-fences which lead to the first counterexample to the $P_{\mathrm{C}}^{n}=P_{\mathrm{LO}}^{n}$ conjecture. This class is invariant. The 3-fence also belongs to the class of Möbius ladders and is denoted $M_{1}$ in Reinelt (1985).

$F_{4}$. The fourth class is the last one defined on fewer than seven nodes. It belongs to the broad class of Möbius ladders. This Möbius ladder is generated by four 3-dicycles and one 4-dicycle. It was named Möbius ladder $M_{2}$ in Reinelt (1985) and is not invariant.

$F_{5}$. This Möbius ladder is generated by three 3-dicycles and two 4-dicycles. It is obtained from the basic Möbius ladder $M_{2}$ by extending a 3-dicycle to a 4-dicycle. The class is not invariant.

$F_{6}$. Here we have another Möbius ladder generated by three 3-dicycles and two 4-dicycles. It is also obtained from $M_{2}$ by extending a 3-dicycle to a 4-dicycle. The class is invariant.

$F_{7}$. This Möbius ladder is generated by six 3-dicycles and one 4-dicycle. It can be constructed from $M_{2}$ by adding two 3-dicycles. The class is not invariant.

$F_{8}$. This is a Möbius ladder generated by five 3-dicycles and two 4-dicycles. The class is not invariant. (Note that this Möbius ladder is not obtained from another one by adding two 3-dicycles.)

$F_{9}$. Adding two 3-dicycles in yet another way to $M_{2}$ leads to this Möbius ladder generated by six 3-dicycles and one 4-dicycle. The class is not invariant.

$F_{10}$. A Möbius ladder generated by three 4-dicycles and two 3-dicycles. It is obtained from the basic Möbius ladder $M_{1}$ by adding two 3-dicycles. The class is not invariant.

$F_{11}$. This Möbius ladder is generated by seven 3-dicycles. It is named $M_{3}$ in Reinelt (1985) and is invariant.

$F_{12}$. This is the Möbius ladder $M_{4}$ (see Reinelt, 1985). It is generated by seven 3-dicycles and it is invariant.

$F_{13}$. This is yet another Möbius ladder generated by seven 3-dicycles. The digraph is invariant.

$F_{14}$. This digraph is known as 3-wheel (Reinelt, 1985) or augmented 3-fence (Suck, 1991). The two names correspond to two different possible generalizations. The class is invariant.

$F_{15}$. A Möbius ladder generated by eight 3-dicycles and one 4-dicycle. The digraph is not invariant. 


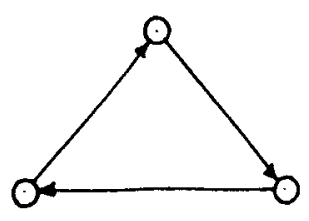

$F_{2}$

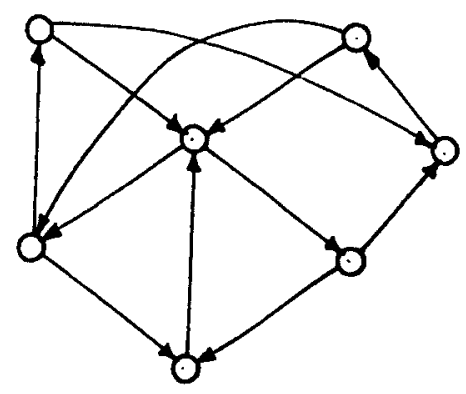

$F_{3}$

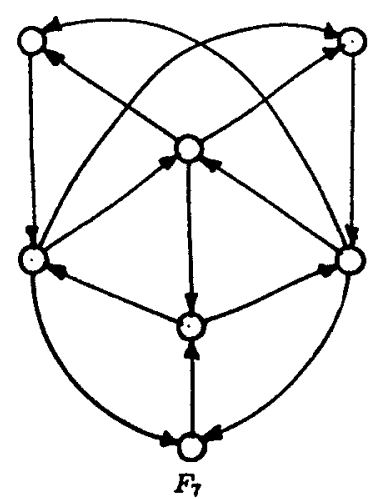

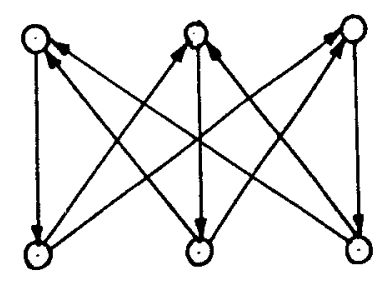

$F_{3}$

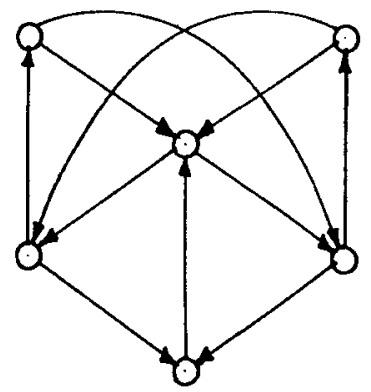

$F_{4}$
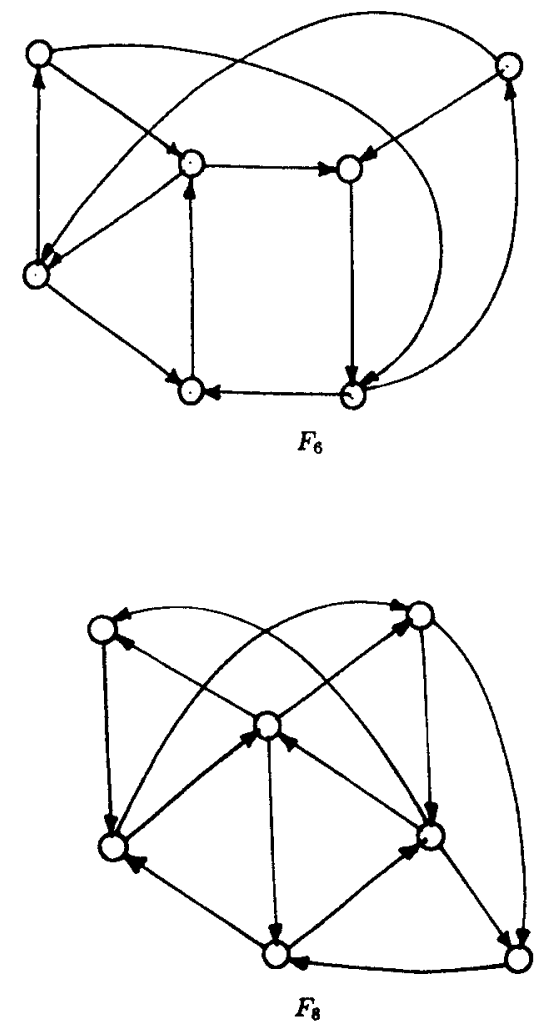

Fig. 4.1. Facets of $P_{\mathrm{LO}}^{7}$ 


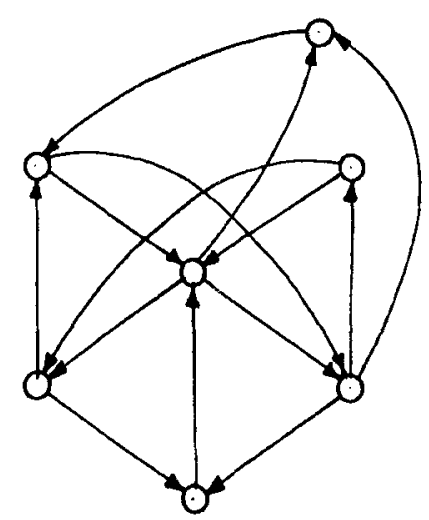

$F_{9}$

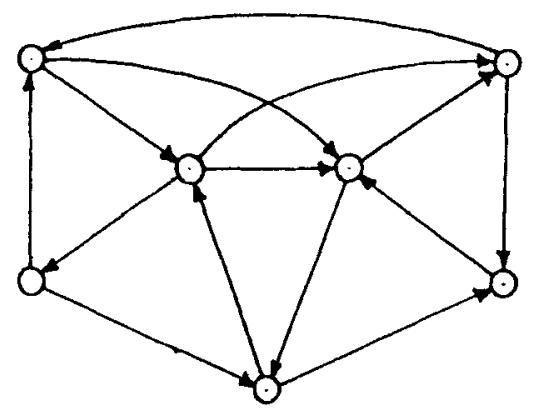

$F_{11}$

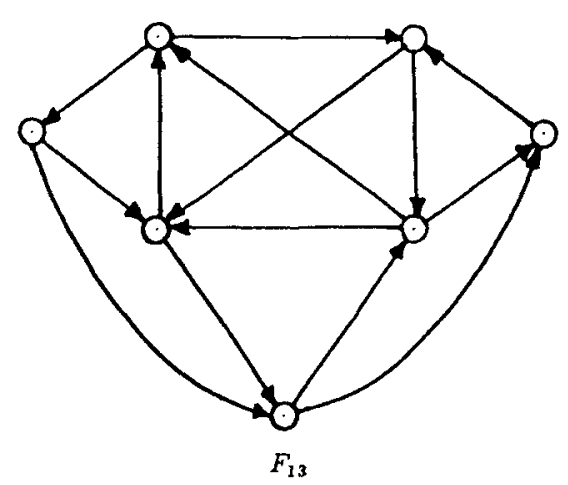

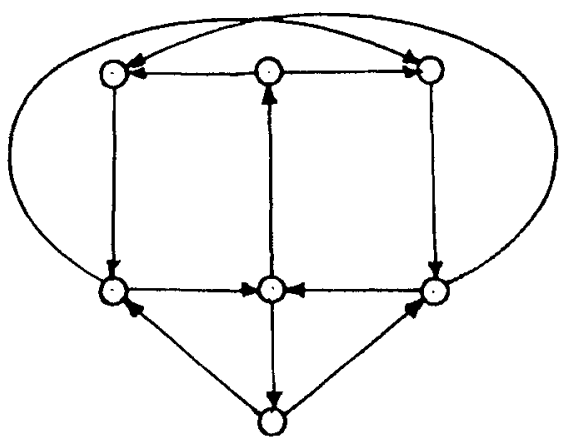

$F_{10}$

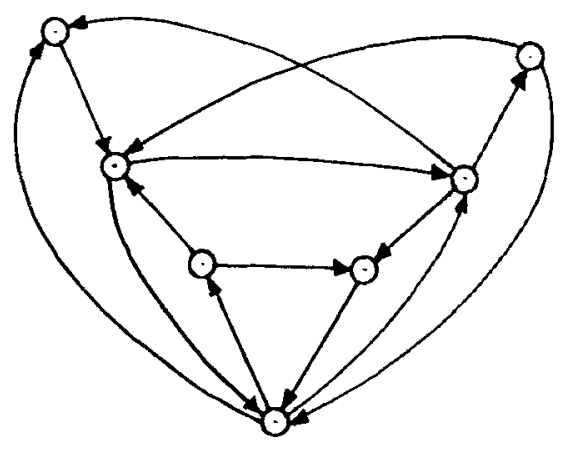

$F_{12}$

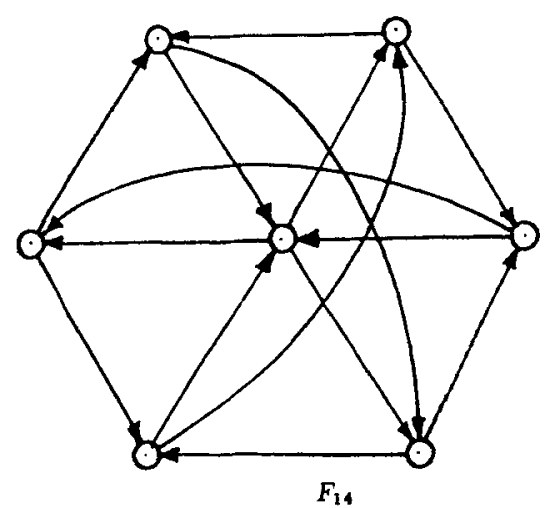

Fig. 4.1 (continued) 
G. Reinelt
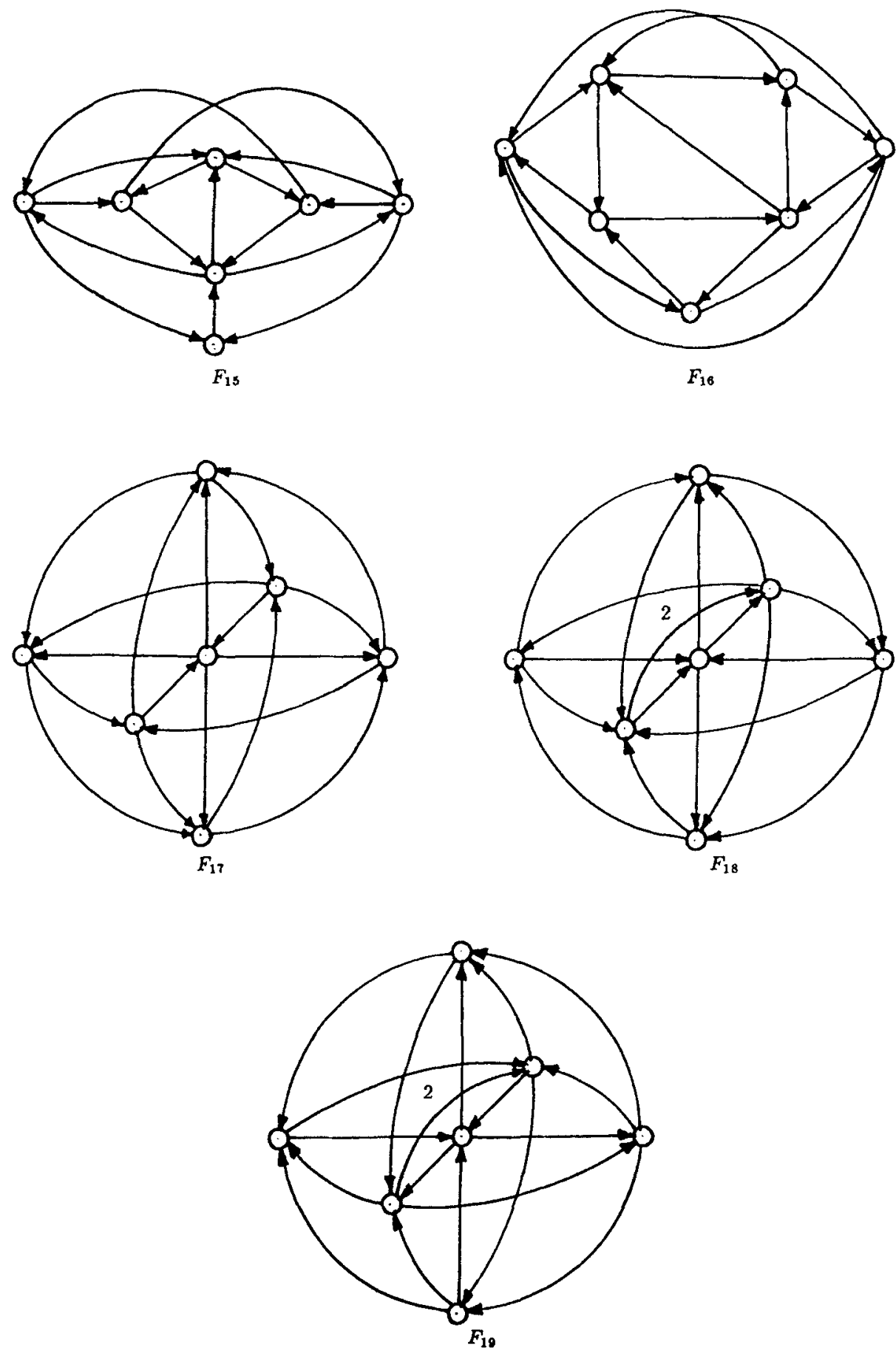

Fig. 4.1 (continued) 
Table 4.1. The polyhedral structure of $P_{\text {LO }}^{7}$.

\begin{tabular}{|c|c|c|c|c|}
\hline Facet & $\begin{array}{l}\text { Number of } \\
\text { vertices on facet }\end{array}$ & Invariant & $\begin{array}{l}\text { Number of } \\
\text { different facets }\end{array}$ & $\begin{array}{l}\text { Right-hand } \\
\text { side }\end{array}$ \\
\hline$F_{1}$ & 2,520 & Yes & 42 & 1 \\
\hline$F_{2}$ & 2,520 & Yes & 70 & 2 \\
\hline$F_{3}$ & 126 & Yes & 840 & 7 \\
\hline$F_{4}$ & 126 & No & 5,040 & 8 \\
\hline$F_{5}$ & 67 & No & 10,080 & 9 \\
\hline$F_{6}$ & 44 & Yes & 5,040 & 9 \\
\hline$F_{7}$ & 104 & No & 5,040 & 10 \\
\hline$F_{8}$ & 67 & No & 10,080 & 10 \\
\hline$F_{9}$ & 67 & No & 10,080 & 10 \\
\hline$F_{10}$ & 44 & No & 5,040 & 9 \\
\hline$F_{11}$ & 67 & Yes & 5,040 & 10 \\
\hline$F_{12}$ & 104 & Yes & 5,040 & 10 \\
\hline$F_{13}$ & 67 & Yes & 5,040 & 10 \\
\hline$F_{14}$ & 126 & Yes & 840 & 10 \\
\hline$F_{15}$ & 104 & No & 5,040 & 11 \\
\hline$F_{16}$ & 104 & Yes & 5,040 & 11 \\
\hline$F_{17}$ & 28 & No & 2,520 & 13 \\
\hline$F_{18}$ & 28 & Yes & 2,520 & 14 \\
\hline$F_{19}$ & 28 & Yes & 5,040 & 14 \\
\hline
\end{tabular}

$F_{16}$. An invariant Möbius ladder generated by nine 3-dicycles.

$F_{17}$. The facet associated with this digraph was not known before. The class is not invariant.

$F_{18}$. This is another example of a new class of facet-defining inequalities and the first one whose coefficients are not all 1 (i.e., it is not a rank facet). The class is invariant.

$F_{19}$. This is another facet with coefficient 2 . It has the same support as $F_{18}$ (if arc directions are left out of consideration). The class is invariant.

The discussion shows that the concept of Möbius ladders provides a very rich class of facet-defining inequalities. Therefore, though never stated explicitly, all facets of $P_{\text {LO }}^{7}$ except for $F_{17}, F_{18}$, and $F_{19}$ were already implicitly known. We were able to generalize $F_{17}$ and $F_{18}$ to derive new facets of $P_{\text {LO }}^{n}$ for $n>7$. Our attempts to generalize $F_{19}$ failed so far.

If we count the digraphs obtained by arc reversal as new graphs we have therefore 27 different graphs which yield facets of $P_{\text {Lo }}^{7}$. Table 4.1 summarizes some properties of the 19 classes. It gives the number of vertices on each facet of the respective class, the number of different facets in each class, the right-hand side of the associated inequality, and states whether we have invariance or not.

Table 4.1 also gives an indication on the importance of trivial and 3-dicycle inequalities since each such facet contains half of the vertices of $P_{10}^{7}$. The usefulness of these inequalities has also been observed in practical computations (Grötschel et al. 1984; Reinelt, 1985). 
Summing up we obtain that the total number of facets of $P_{L 0}^{7}$ is at least 87,472 . If the hypothesis is true that on every facet of $P_{\text {Lo }}^{7}$ there are two vertices corresponding to linear orderings which differ only in two adjacent positions, then this number gives the total number of facets of $P_{L O}^{7}$. It should be noted that there is a high chance for this hypothesis to be true. In the case of the symmetric traveling salesman problem on eight nodes it would have been sufficient to consider just one ray corresponding to a two-exchange (i.e., replacement of two edges by two different edges to form a new tour) to derive the complete description of the associated polytope.

\section{References}

V. J. Bowman (1972), Permutation polyhedra, SIAM J. Appl. Math. 22, 580-589.

J. S. de Cani (1969), Maximum likelihood paired comparison ranking by linear programming, Biometrika 56, 537-545.

T. Christof (1991), Ein Verfahren zur Transformation zwischen Polyederdarstellungen, Diplomarbeit, Universität Augsburg.

T. Christof, M. Jünger, and G. Reinelt (1991), A Complete Description of the Traveling Salesman Polytope on 8 Nodes, Oper. Res. Lett. 10, 497-500.

T. Dridi (1980), Sur les distribution binaires associees a des distributions ordinales, Math. Sci. Humaines 69, $15-31$.

P. C. Fishburn (1990), Binary probabilities induced by rankings, SIAM J. Discrete Math. 3, 478-488.

P. C. Fishburn (1991), Induced Binary Probabilities and the Linear Ordering Polytope: A Status Report, AT\&T Bell Laboratories.

M. Grötschel, M. Jünger, and G. Reinelt (1984), A cutting plane algorithm for the linear ordering problem, Oper. Res. 32, 1195-1220.

M. Grötschel, M. Jünger, and G. Reinelt (1985), Facets of the linear ordering polytope, Math. Programming 33, 43-60.

D. Hausmann (1980), Adjacency on Polytopes in Combinatorial Optimization, Hain, Meisenheim.

J. Leung and J. Lee (1990), Reinforcing Old Fences Gives New Facets, Report, Yale University.

G. Reinelt (1985), The Linear Ordering Problem: Algorithms and Applications, Research and Exposition in Mathematics, Vol. 8, Heldermann-Verlag, Berlin.

R. Suck (1991), Geometric and Combinatorial Properties of the Polytope of Binary Choice Probabilities, Report, Universität Osnabrück.

S. N. Tschernikov (1971), Lineare Ungleichungen, Deutscher Verlag der Wissenschaften, Berlin.

H. P. Young (1978), On permutations and permutation polytopes, Math. Programming Stud. 8, $128-140$.

Received January 2, 1992.

Note added in proof. Using a similar computer code, R. Euler and H. Le Verge (Universite de Bretagne Occidentale, Brest, France) could show that the description of $P_{\text {Lo }}^{\gamma}$ given here is indeed complete. 